\title{
Material-Enhanced Laser \\ Desorption/Ionization (MELDI) - A New \\ Protein Profiling Tool Utilizing Specific \\ Carrier Materials for Time of Flight \\ Mass Spectrometric Analysis
}

\author{
Isabel Feuerstein, ${ }^{*}$ Muhammad Najam-ul-Haq,, Matthias Rainer, ${ }^{*}$ \\ Lukas Trojer, ${ }^{*}$ Rania Bakry, Nurul Hidayat Aprilita, Günther Stecher, \\ Christian W. Huck, and Günther K. Bonn \\ Institute of Analytical Chemistry and Radiochemistry, Leopold Franzens University, Innsbruck, Austria
}

Helmut Klocker and Georg Bartsch

Department of Urology, Innsbruck Medical University, Innsbruck, Austria

\section{Andras Guttman}

Horváth Laboratory of Bioseparation Sciences, Innsbruck, Austria

Over the past couple of years, proteomics pattern analysis has emerged as an effective method for the early diagnosis of diseases such as ovarian, breast, or prostate cancer, without identification of single biomarkers. MALDI-TOF MS, for example, offers a simple approach for fast and reliable protein profiling, especially by using carrier materials with various physical and chemical properties, in combination with a MALDI matrix. This approach is referred to as materialenhanced laser desorption/ionization (MELDI). In this paper, we report the development and application of derivatized carrier materials [cellulose, silica, poly(glycidyl methacrylate/divinylbenzene) (GMA/DVB) particles, and diamond powder] for fast and direct MALDI-TOF MS protein profiling. The applicability of MELDI for rapid protein profiling was evaluated with human serum samples. These carriers, having various hydrophobicities, resulted in characteristic mass fingerprints, even if all materials were derivatized with iminodiacetic acid (IDA) to yield an immobilized metal affinity chromatography (IMAC) functionality. Our study demonstrates that analyzing complex biological samples, such as human serum, by employing different MELDI carrier materials yielded type- and size-dependent performance variation. (J Am Soc Mass Spectrom 2006, 17, 1203-1208) (c) 2006 American Society for Mass Spectrometry

$\mathrm{T}$ The quest to diagnose and identify diseases at early stages depends mainly on the accurate determination of the marker entities in the biological fluids. These marker entities are normally very low concentrated [1], and highly sensitive methods are required to bind them effectively from the biosamples and to measure them with matrix-assisted laser desorption/ionization time of flight mass spectrometry (MALDI-TOF MS) [2]. MALDI mass spectrometry, in recent times, has proven to be an authentic technique to analyze peptides and pro-

Published online June 12, 2006

Address reprint requests to Dr. I. Feuerstein, Institute of Analytical Chemistry and Radiochemistry, Leopold Franzens University, Innrain 52a, 6020Innsbruck, Austria. E-mail: Isabel.Feuerstein@uibk.ac.at

* These authors contributed equally to this work. teins $[3,4]$. These biomolecules out of the complex biofluids like serum are difficult to ionize and thus, bring into time of flight (TOF) because of the complexity of the sample and the suppressing nonvolatile salts [5]. The aforementioned problems are tackled by another technique introduced years ago, namely, surface-enhanced laser desorption/ionization time of flight mass spectrometry (SELDI-TOF MS), for the detection of several peptides and proteins from complex biological specimens [6-8]. This affinity-based method utilizes selective adsorption of proteins on various chromatographic surfaces to reduce sample complexity $[9,10]$. Spectra generated by SELDITOF MS usually show limited peak and binding capacity due to the smaller surface area of the plane protein chip [11]. Limitations of SELDI are also associated with the lower mass resolution of the applied MS instrument that 
has made it difficult to reproducibly detect protein patterns and identify individual proteins in complex mixtures [12-14].

In this paper, we describe a material-based approach referred to as material-enhanced laser desorption/ionization (MELDI) that utilizes various functionalized carrier materials such as cellulose, silica, poly(GMA/DVB) particles, and diamond powder for direct protein profiling employing a MALDI-TOF MS instrument. Due to the high surface area, different physical characteristics and binding capacities of the investigated materials, this method allows detection of a large number of peptides and proteins within a broad molecular mass range.

\section{Experimental}

\section{Materials}

Iminodiacetic acid (IDA) was purchased from Sigma Aldrich (St. Louis, MO). Silica particles (Grom-SIL $100 \mathrm{~nm}$ and $30 \mathrm{~nm}$ ) were from Grom Analytik (Rottenburg-Hailfingen, Germany), spherical cellulose (Celluflow C-25) from Collaborative Laboratories (New York, NJ), fibrous cellulose from Sigma Aldrich (St. Louis, MO), and diamond powder from Ceratonia (Eltmann, Germany). Protein calibration standard-I was purchased from Bruker Daltonics (Bremen, Germany). Serum samples were provided by the Department of Urology at the Medical University of Innsbruck, Austria.

\section{Particle Preparation and Derivatization}

Cellulose particles. Cellulose was treated with glycidyl methacylate (Figure 1a) at elevated temperature in the presence of a redox catalyst as described in $[15,16]$.

Silica particles. After purification and activation [17], silica gel was subjected to surface modification with glycidoxypropyltrimethoxysilane (GLYMO) in dry toluene in the presence of triethylamine (Figure 1b) [18].

GMA/DVB-polymer. Glycidyl methacrylate (GMA) was copolymerized with divinylbenzene (DVB) (Figure 1c) by free radical polymerization in the presence of $\alpha, \alpha^{\prime}$-azoisobutyronitrile (AIBN) as thermal initiator [19, 20]. The precipitation polymerization was performed in $\mathrm{ACN}$ and yielded strongly crosslinked beads featuring epoxide groups on the surface.

All mentioned particles were then derivatized with IDA under alkaline conditions. After filtration and several washing steps, the products were loaded with $\mathrm{Cu}^{2+}$ by incubation with $50 \mathrm{mM} \mathrm{CuSO}_{4}$ solution for $2 \mathrm{~h}$ at room temperature (RT).

Diamond powder. Diamond powder was first oxidized to form a surface oxide layer by means of a mixture of sulfuric acid and nitric acid (9:1) for $24 \mathrm{~h}$ (Figure 1d). After centrifugation, the solid oxidized diamond pow- der was washed with $0.1 \mathrm{M} \mathrm{NaOH}$, with $0.1 \mathrm{M} \mathrm{HCl}$, and finally with water. The resulting carboxylated diamond powder was treated with thionyl chloride $(10 \mathrm{~mL})$ at $50{ }^{\circ} \mathrm{C}$ for $24 \mathrm{~h}$. The excess thionyl chloride was removed by rotary vacuum evaporator, followed by washing with dry THF [21]. IDA was bound to the generated acid chloride by nucleophilic attack of the imino-N on the carbonyl group in alkaline condition. The final product was vacuum filtered, washed, and loaded with $\mathrm{Cu}^{2+}$ by incubation with $50 \mathrm{mM} \mathrm{CuSO}_{4}$ solution for $2 \mathrm{~h}$ at RT.

\section{Sample Preparation}

Forty $\mu \mathrm{l}$ serum was treated with $30 \mu \mathrm{l} 8 \mathrm{M}$ urea containing 1\% 3-[(3-cholamidopropyl)dimethylammonio]-1-propanesulfonate (CHAPS) in phosphate buffered saline (PBS) by shaking for a few min. Afterwards, $100 \mu \mathrm{l} 1 \mathrm{M}$ urea containing $0.125 \%$ CHAPS was added and shaken briefly again. This mixture was diluted to $850 \mu \mathrm{l}$ with PBS and vortexed at $4{ }^{\circ} \mathrm{C}$ for $10 \mathrm{~min}$. Three $\mathrm{mg}$ of each $\mathrm{Cu}^{2+}$ loaded material was separately filled in $0.5 \mathrm{ml}$ micro centrifuge tubes, activated with $50 \mathrm{mM}$ sodium acetate buffer $(\mathrm{pH}=4.0)$ for $5 \mathrm{~min}$ at $\mathrm{RT}$, and centrifuged at 13,000 rpm for $1 \mathrm{~min}$. The supernatant was discarded and the residue was equilibrated twice with $200 \mu \mathrm{l}$ of PBS buffer $(\mathrm{pH}=7.4)$. Four hundred $\mu \mathrm{l}$ diluted serum sample was added after the equilibration step and the whole suspension mixture was incubated on a platform shaker at $1500 \mathrm{rpm}$ for $2 \mathrm{~h}$ at $30^{\circ} \mathrm{C}$. To remove unbound proteins, the sample-treated carrier materials were washed three times with $200 \mu$ l equilibration buffer, followed by a quick washing step with $200 \mu \mathrm{l}$ deionized water. One $\mu \mathrm{l}$ serum-material mixture was spotted on a stainless steel target plate (MTP 384 ground steel, Bruker Daltonics, Bremen, Germany) followed by the addition of $1 \mu \mathrm{l}$ saturated sinapinic acid solution ( $50 \%$ ACN $/ 0.1 \%$ TFA).

\section{MALDI-TOF MS System}

Proteins bound to the derivatized carrier materials were analyzed by MALDI-TOF MS (Ultraflex MALDI-TOFTOF MS, Bruker Daltonics) in linear mode at a detector energy of $1623 \mathrm{~V}$. All spectra were recorded by summation of 270 laser shots in the mass range of $\mathrm{m} / \mathrm{z} 2000$ to 18,000 . For data validation, external calibration was performed with a standard mixture containing insulin, ubiquitin, cytochrome $c$, and myoglobin. Flex control V 2.0 was used for acquisition and all further data processing was carried out using Flex analysis V 2.0.

\section{Results and Discussion}

\section{Reproducibility}

One of the main benefits of MELDI is its utilization of different materials with high capacity and surface functionality to bind serum constituents. To investigate the 


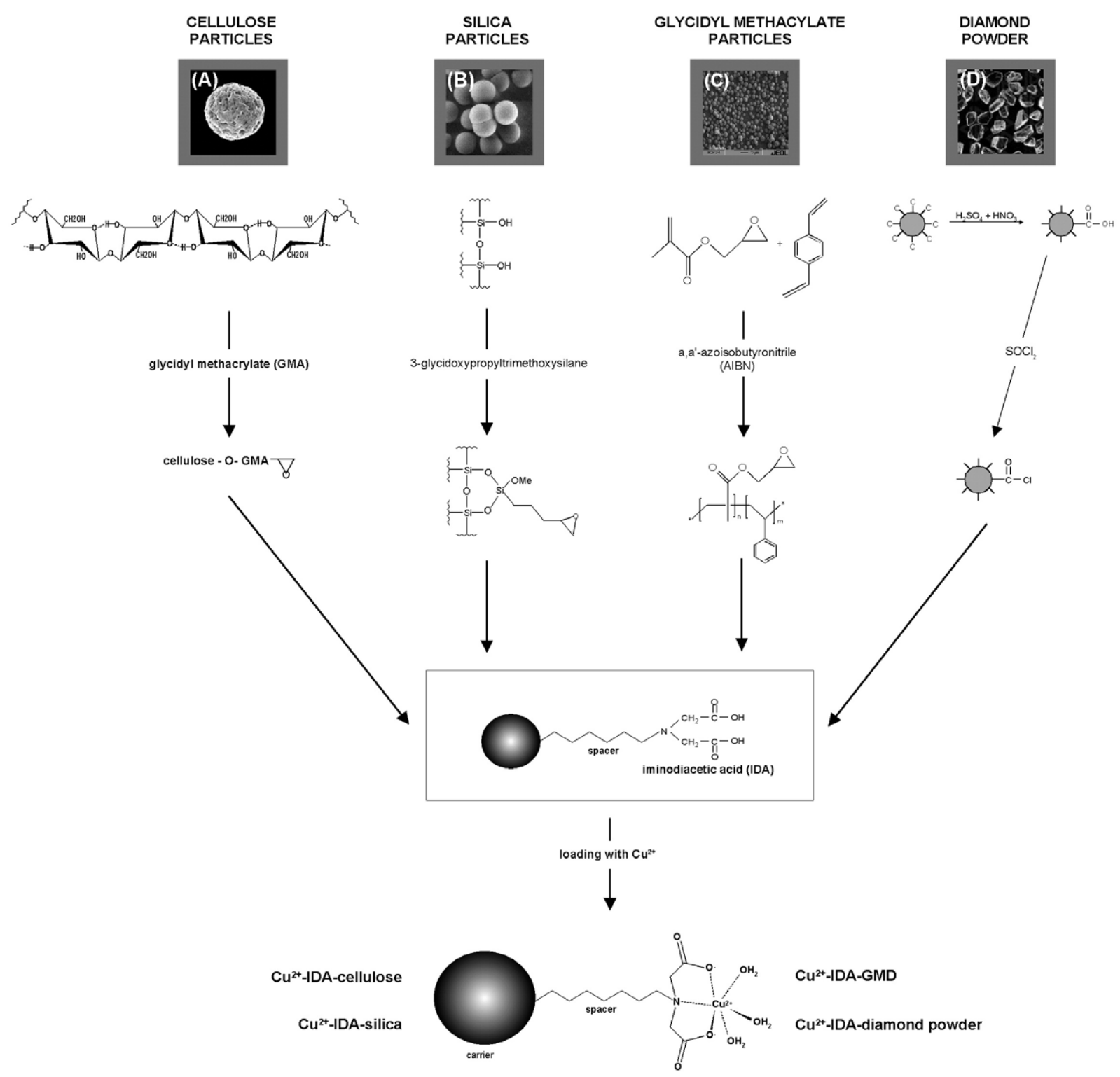

Figure 1. Reaction scheme for MELDI material synthesis. Derivatization procedure for preparation of iminodiacetic acid (IDA) linked cellulose (a), silica (b) poly(GMA/DVB) particles (c), and diamond powder (d).

applicability of MELDI, these materials were checked for reproducibility of derivatization and sample preparation with human serum samples. The spectra recorded for every material showed similar serum mass fingerprints with only minor intensity differences, which can be attributed to the variation resulting from sample preparation in suspension, preparation on MALDI target, and matrix crystallization effects. Furthermore, statistical tests were also applied by choosing the most intense peaks out of four mass spectra recorded with the same human serum sample at different times on each material. The selected mass peaks, along with their standard deviation, are shown in Table 1. Detailed description on reproducibility and robustness using cellulose or silica particles was recently reported elsewhere [16, 22].

\section{MELDI Carrier Materials}

During the evaluation process of various functionalized carrier materials, we found that not all of them were equally suitable for MELDI. More precisely, their applicability strongly depended on their physical properties such as particle shape as well as particle pore size. Figure 2 depicts the effect of the shape of cellulose particles on MELDI-TOF-MS analysis. For that purpose human serum sample was mixed with derivatized fibrous and spherical cellulose particles and subjected to direct MALDI-TOF MS analysis (Figure 2). As it can be observed, cellulose carrier materials of different shapes resulted in unique peak patterns, being the $8-10$ $\mu \mathrm{m}$ size range the most effective, in the sense of number and signal intensity of the resulting peaks. As the 
Table 1. Selected $\mathrm{m} / \mathrm{z}$ values of four independent measurements showing the reproducibility of serum protein profiling using various copper loaded MELDI materials.

\begin{tabular}{|c|c|c|c|c|c|}
\hline & $m / z$ & $m / z$ & $m / z$ & $m / z$ & $m / z$ \\
\hline \multicolumn{6}{|l|}{ Cellulose particles } \\
\hline MS pattern 1 & 3271.61 & 3369.81 & 5337.78 & 5906.37 & 8138.82 \\
\hline MS pattern 2 & 3273.69 & 3372.62 & 5337.57 & 5905.28 & 8141.27 \\
\hline MS pattern 3 & 3270.31 & 3369.32 & 5337.47 & 5904.43 & 8141.51 \\
\hline MS pattern 4 & 3271.85 & 3371.05 & 5336.97 & 5905.34 & 8143.23 \\
\hline Standard Deviation & 1.39 & 1.47 & 0.35 & 0.79 & 1.81 \\
\hline \multicolumn{6}{|l|}{ Silica particles } \\
\hline MS pattern 1 & 2662.68 & 3179.05 & 4577.04 & 5073.99 & 6650.74 \\
\hline MS pattern 2 & 2663.14 & 3178.66 & 4577.76 & 5073.49 & 6650.04 \\
\hline MS pattern 3 & 2664.43 & 3179.35 & 4578.24 & 5071.86 & 6645.31 \\
\hline MS pattern 4 & 2660.55 & 3176.40 & 4576.62 & 5071.60 & 6651.43 \\
\hline Standard Deviation & 1.61 & 1.34 & 0.72 & 1.19 & 2.77 \\
\hline \multicolumn{6}{|l|}{ GMA/DVB particles } \\
\hline MS pattern 1 & 2023.54 & 2663.89 & 3197.86 & 5349.32 & 8622.78 \\
\hline MS pattern 2 & 2025.52 & 2666.67 & 3199.76 & 5352.66 & 8625.36 \\
\hline MS pattern 3 & 2024.65 & 2664.78 & 3198.37 & 5351.05 & 8625.72 \\
\hline MS pattern 4 & 2024.02 & 2664.38 & 3198.97 & 5351.56 & 8624.95 \\
\hline Standard Deviation & 0.86 & 1.22 & 0.82 & 1.39 & 1.32 \\
\hline \multicolumn{6}{|l|}{ Diamond Powder } \\
\hline MS pattern 1 & 3312.25 & 4149.52 & 5902.05 & 6628.91 & 7764.36 \\
\hline MS pattern 2 & 3311.73 & 4149.91 & 5903.21 & 6628.35 & 7763.71 \\
\hline MS pattern 3 & 3312.91 & 4148.02 & 5902.98 & 6629.05 & 7764.52 \\
\hline MS pattern 4 & 3310.85 & 4149.01 & 5903.37 & 6628.74 & 7762.98 \\
\hline Standard Deviation & 0.87 & 1.19 & 0.59 & 0.79 & 1.14 \\
\hline
\end{tabular}

particle size increased to $20-30 \mu \mathrm{m}$, or the shape changed from spherical to fibrous, the efficiency of the carrier decreased tremendously, suggesting that fibrous cellulose and large spherical cellulose particles $(>10$ $\mu \mathrm{m})$ are not adequately suitable for MELDI. Furthermore, the porosity of the investigated MELDI supports influenced the binding properties and appearance of the resulting mass. Pore size has thus to be carefully selected, as porosity has great impact on the desorption process and consequently on the mass spectra obtained. As described earlier by Trojer et al. [22], narrow pore silica particles $(6 \mathrm{~nm})$ did not provide adequate MS traces. These results suggest that particles with only certain properties, i.e., highly porous, spherical, and in the low $\mu \mathrm{m}$ size range, can be effectively utilized in MELDI.

\section{Hydrophilic and Hydrophobic Interactions}

Human serum samples were used to check the bound and adsorbed proteins in the mass range of $\mathrm{m} / \mathrm{z} 2000$ to 18,000 , to demonstrate the applicability of the four carrier materials investigated in MELDI-TOF MS. Aliquots of a human serum sample were mixed with each of the four carrier materials, followed by sample preparation and TOF MS analysis as described in the Experimental section. The MS traces in Figure 3a illustrate the representative MELDI spectra for each carrier material in the order of increasing hydrophobicity (cellulose, silica, GMA/DVB particles, and diamond powder [23]. Due to the same surface functionality used for all carrier materials (copper loaded IDA), some peaks in the resulting mass fingerprints exhibited the same $\mathrm{m} / \mathrm{z}$ values, such as the 4160, 6625, and 15,149 peaks (see asterisks in Figure 3a). However, upon closer examination of the individual profiles in the respective MELDI spectra, noteworthy differences were found. The mass ranges of the most interesting sections are enlarged in Figure $3 b$, panels $1-5$. The very left panel (1) depicts the mass range $m / z 7400$ to 8300 , emphasizing some spectral similarities between cellulose and GMA/DVB polymer while diamond and silica carrier materials gave different MELDI spectra in this mass range. Panel 2 in Figure $3 \mathrm{~b}$ compares the spectra in the $\mathrm{m} / \mathrm{z}$ range 8500 to 9600 , revealing significant differences among the effects of the various carrier materials, showing particularly weak performance for cellulose and silica particles, probably due to their rather hydrophilic nature.

MELDI spectra delineating the $\mathrm{m} / \mathrm{z}$ range 14,900 to 15,400 (Figure 3b, panel 3) using copper loaded carrier materials exhibited three consistent peaks at distinct positions (see asterisks). Actually, the absence and presence of some peaks depicted in panels $3-5$ emphasize the complementarity of these four carrier materials of increasingly hydrophobic properties. For example, the trace corresponding to diamond powder carrier material in panel 5 exhibits three distinct peaks between $\mathrm{m} / \mathrm{z} 17,000$ and 17,700 , whereas in the other spectra acquired using copper loaded spherical cellulose, silica particles, and GMA/DVB particles, no peaks were detectable. However, it is apparent from Figure 3 that a significant number of proteins bound to these carrier 


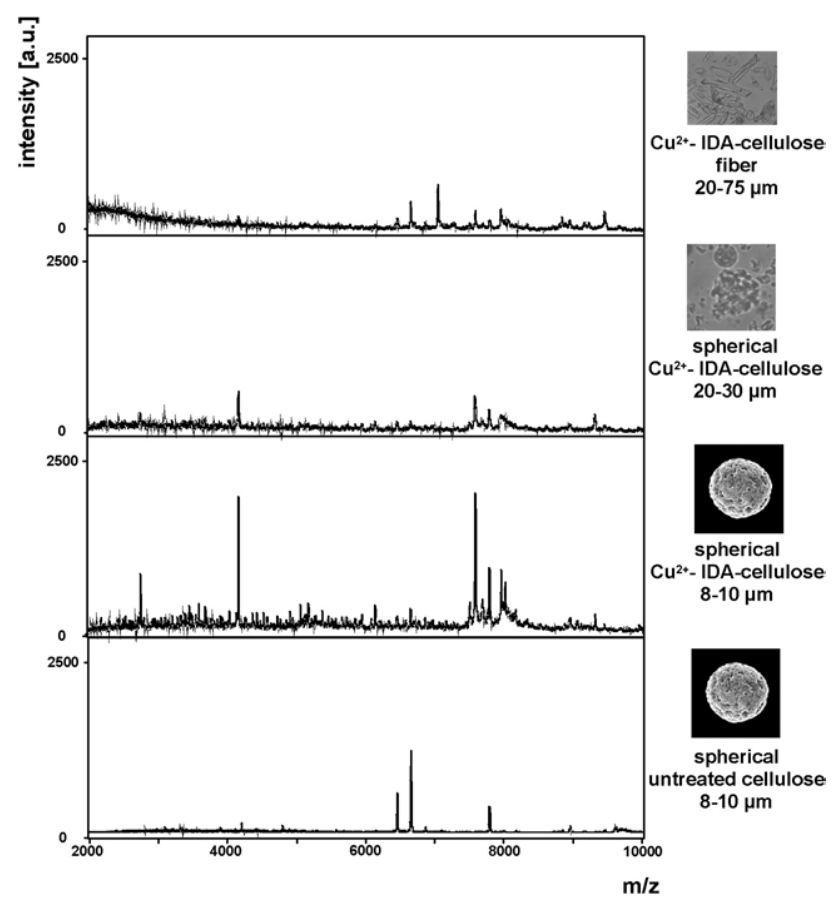

Figure 2. MELDI-TOF-MS based protein profiling using various shaped $\mathrm{Cu}^{2+}$-IDA-celluloses. Human serum sample was analyzed using derivatized and copper loaded fibrous $(20-75 \mu \mathrm{m})$ and spherical $(20-30 \mu \mathrm{m}$ and $8-10 \mu \mathrm{m})$ cellulose particles as well as untreated and underivatized spherical cellulose particles (8-10 $\mu \mathrm{m})$ to show the influence of particle size, shape, and derivatization on the MELDI process.

materials, probably because of the adaptable range of hydrophilicity and hydrophobicity.

Summarizing, the use of MELDI will increase the number of quality data derived from biological samples at the peptide and protein level. It will have a major impact on basic biomedical research as well as on discovery of new drug targets and diagnostic markers. These carrier materials can simultaneously provide high-affinity sites to bind multitude of proteins in a very short period of time by employing the same buffer systems for sample preparation. In addition, these materials also allow analysis of captured proteins directly on the target. The diversity in physical and chemical characteristics, e.g., shape and porosity as well as hydrophobic and hydrophilic character of the derivatized materials, were responsible for the improved sensitivity, specificity, capacity, and broad range of information content of the resulting spectra.

\section{Efficiency Enhancement of MELDI Methodology}

To assess the derivatization mediated efficiency enhancement in MELDI, underivatized cellulose particles were used for serum sample preparation (lower panel, Figure 2). The results clearly showed performance enhancement due to IDA derivatization. The TOF-MS spectra using untreated spherical cellulose carrier material provided very simple profiles with just a few evaluable peaks. The copper loaded IDA linked material, on the other hand, exhibited a complex peak pattern useful for protein profiling from human serum samples.

To further demonstrate the power of MELDI methodology, an untreated raw serum (the same used for all of the other experiments) was analyzed with conventional MALDI sample preparation. In comparison with conventional MALDI, the presented MELDI sample preparation clearly proved the power of our approach. Due to high affinity and high capacity of MELDI materials, all recorded spectra resulted in more intense MS traces than the spectra obtained by conventional MALDI sample preparation (data not shown). As already mentioned in the introduction, complex biofluids like serum are hard to analyze by mass spectrometric based methods, especially because of their complexity and suppressing nonvolatile salts. Therefore, before mass spectrometric analysis, special pretreatment is needed. The presented approach not only employs the developed chemistry, which reduces the complexity of the sample, but also the inherited physical characteristics of the materials, e.g., high surface area, which

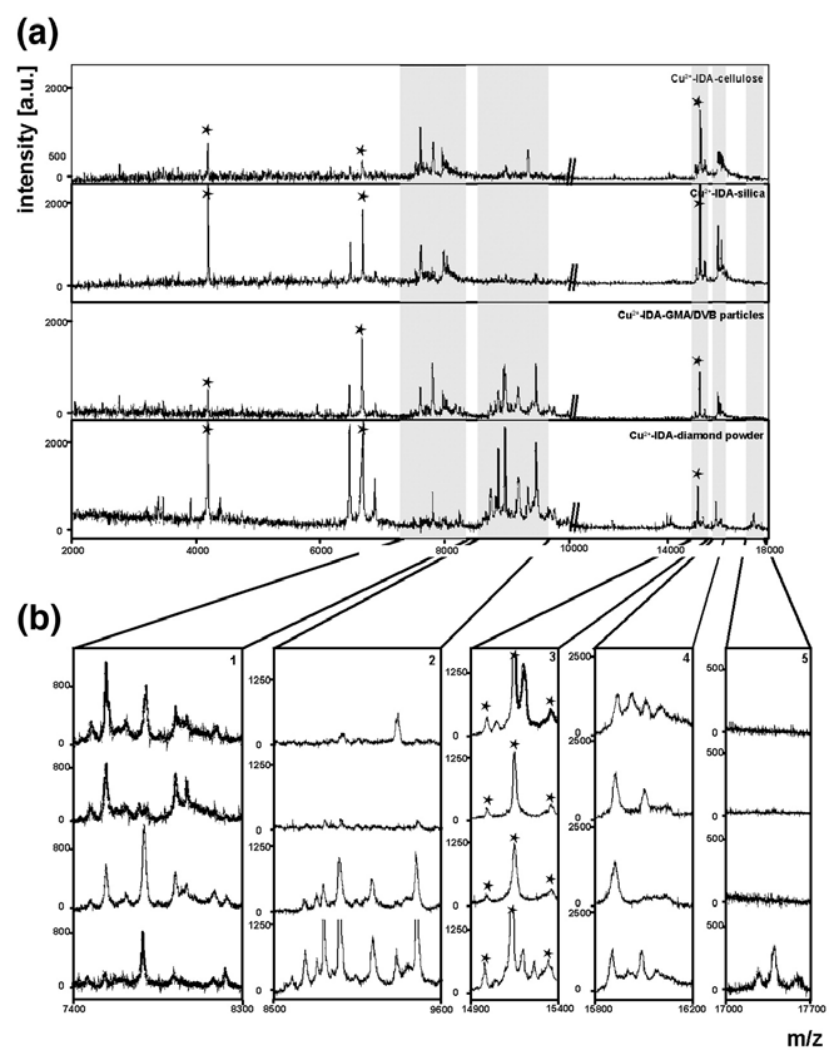

Figure 3. MELDI spectra using different copper loaded materials. Spectra were recorded in $\mathrm{m} / \mathrm{z}$ range from 2000 to 18,000 . Human serum samples were analyzed by binding to spherical cellulose, silica and poly(GMA/DVB) particles as well as to diamond powder (a). Extended MELDI spectra of different mass regions (b): Sections of 7400 to 8300 (1), from 8500 to 9600 (2), from 14,900 to 15,400 (3), from 15,800 to 16,200 (4), and from 17,000 to $17,700(5)$. 
enables the enrichment of proteins and peptides, convey the characteristic protein profiles.

\section{Conclusions}

The introduction of MELDI as a profiling technique provides a sensitive, multiplexed protein pattern analysis approach offering accurate and reproducible MStraces that can be useful for wide ranging applications. The recorded spectra have been proven to be specific for each carrier material. The ongoing development of an automated MELDI technique employing liquid handling robots devices for high-throughput analysis of clinical relevant samples particularly represents a key towards multiplexed protein profiling.

\section{References}

1. Merrell, K.; Southwick, K.; Graves, S. W.; Esplin, M. S.; Lewis N. E.; Thulin, C. D. Analysis of Low-Abundance, Low-Molecular-Weight Serum Proteins Using Mass Spectrometry. J. Biomol. Tech. 2004, 15, 238-248.

2. Hillenkamp, F.; Karas, M.; Beavis, R. C.; Chait, B. T. Matrix-Assisted Laser Desorption Ionization Mass-Spectrometry of Biopolymers. Anal. Chem. 1991, 63, 1193A-1202A.

3. Toshifumi, T. Proteomics Research by Matrix-Assisted Laser Desorption/Ionization Mass Spectrometry. Reza. Kenkyu 2003, 31, 13-15.

4. Wang, R.; Chait, B. T. High-Accuracy Mass Measurement as a Tool for Studying Proteins. Curr. Opin. Biotechnol. 1994, 5, 77-84.

5. Brockman, A. H.; Dodd, B. S.; Orlando, R. A Desalting Approach for MALDI-MS Using On-Probe Hydrophobic Self-Assembled Monolayers. Anal. Chem. 1997, 69, 4716-4720.

6. Adam, B.-L.; Vlahou, A.; Semmes, O. J.; Wright, G. L., Jr. Proteomic Approaches to Biomarker Discovery in Prostate and Bladder Cancers. Proteomics 2001, 1, 1264-1270.

7. Tang, N.; Tornatore, P.; Weinberger, S. R. Current Developments in SELDI Affinity Technology. Mass Spectrom. Rev. 2003, 23, 34-44.

8. Shahid M.; Ball, G.; Hornbuckle, J.; Holding, F.; Carmichael, J.; Ellis, I.; Ali, S.; Li, G.; McArdle, S.; Creaser, C.; Rees, R. A Prototype Methodology Combining Surface-Enhanced Laser Desorption/Ionization Protein Chip Technology and Artificial Neutral Network Algorithms to Predict the Chemoresponsiveness of Breast Cancer Cell Lines Exposed to Paclitaxel and Doxorubicin Under in Vitro Conditions. Proteomics 2003, 3, 1725-1737.
9. Issaq, H.; Veenstra, T. D.; Conrads, T. P.; Felschow, D. The SELDI-TOF MS Approach to Proteomics: Protein Profiling and Biomarker Identification. Biochem. Biophys. Res. Commun. 2002, 292, 587-592.

10. Fung, E.; Diamond, D.; Simonsesn, A. H.; Weinberger, S. R. The use of SELDI Protein Chip Array Technology in Renal Disease Research. Methods Mol. Med. 2003, 86, 295-312.

11. Villanueva, J.; Philip, J.; Entenberg, D.; Chaparro, C. A.; Tanwar, M. K.; Holland, E. C.; Tempst, P. Serum Peptide Profiling by Magnetic Particle-Assisted, Automated Sample Processing and MALDI-TOF Mass Spectrometry. Anal. Chem. 2004, 76, 1560-1570.

12. Coombes, K. R.; Morris, J. S.; Hu, L.; Edmonson, S. R.; Baggerly, K. A. Serum Proteomics Profiling-a Young Technology Begins to Mature. Nat. Biotechnol. 2005, 23, 291-292.

13. Diamandis, E. P. Analysis of Serum Proteomic Patterns for Early Cancer Diagnosis: Drawing Attention to Potential Problems. J. Natl. Cancer Inst. 2004, 96, 353-356

14. Petricaoin, E. F.; Liotta, L. A. SELDI-TOF Based Serum Proteomics Pattern Diagnostics for Early Detection of Cancer. Curr. Opin. Biotech. 2004, 15, 24-30.

15. Yang, L.; Jia, L.; Zou, H.; Kong, L.; Zhang, Y. Immobilized IDA-Type $\mathrm{Cu}^{2+}$-Chelating Membrane Affinity Chromatography for Purification of Bovine Liver Catalase. Chin. J. Chromatogr. 1997, 15, 292-295.

16. Feuerstein, I.; Rainer, M.; Bernardo, K.; Stecher, G.; Huck, C. W.; Kofler, K.; Pelzer, A.; Horninger, W.; Klocker, H.; Bartsch, G.; Bonn, G. K. Derivatized Cellulose Combined with: A New Tool for Serum Protein Profiling. J. Proteome. Res. 2005, 4, 2320-2326.

17. Larsson, P. O.; Glad, M.; Hansson, L.; Mansson, M. O.; Ohlson, S.; Mosbach, K. High-Performance Liquid Affinity Chromatography. Adv. Chromatogr. 1983, 21, 41-85.

18. Trojer, L.; Stecher, G.; Feuerstein, I.; Lubbad, S.; Bonn, G. K. Characterization and Evaluation of Metal-Loaded Iminodiacetic Acid-Silica of Different Porosity for the Selective Enrichment of Phosphopeptides. J. Chromatogr. A 2005, 1079, 197-207.

19. Zhang, S.; Huang, X.; Yao, N.; Horváth, C. Preparation of Monodisperse Porous Polymethacrylate Microspheres and Their Application in the Capillary Electrochromatography of Macrolide Antibiotics. J. Chromatogr. A 2002, 984, 193-201.

20. Mujawar, S. K.; Kotha, A.; Rajan, C. R.; Ponrathnam, S.; Shewale, J. G. Development of Tailor-Made Glycidylmethacrylate/Divinylbenzene Copolymer for Immobilization of D-Amino Acid Oxidase from Aspergillus Species Strain 020 and Its Application in the Bioconversion of Cephalosporin C. J. Biotechnol. 1999, 75, 11-22.

21. Ushizawa, K.; Sato, Y.; Mitsumori, T.; Machinami, T.; Ueda, T.; Ando, T. Covalent Immobilization of DNA on Diamond and Its Verification by Diffuse Reflectance Infrared Spectroscopy. Chem. Phys. Lett. 2002, 351, 105-108.

22. Trojer, L.; Stecher, G.; Feuerstein, I.; Bonn, G. K. Cu(II)-Loaded Iminodiacetic Acid/Silica Particles for Protein Profiling of Human Serum Samples Using Surface-Enhanced Affinity Capture: Support Porosity Effects. Rapid Commun. Mass Spectrom. 2005, 19, 3398-3404.

23. Pinzari, F.; Ascarelli, P.; Cappelli, E.; Mattei, G.; Giorgi, R. Wettability of HF-CVD diamond films. Diamond Relat. Mater. 2001, 10, 781-785. 